\title{
AN ELECTRON MIRROR AS AN OBJECTIVE LENS OF THE TRANSMISSION ELECTRON MICROSCOPE
}

\author{
Seitkerim Bimurzaev and Yevgeniy Yakushev
}

G. Daukeev Almaty University of Power Engineering and Telecommunications, Almaty, Kazakhstan

It is known [1] that the resolution of the electron microscope is theoretically limited by large aberrations inherent to electronic lenses with rotational symmetry. The presence of these aberrations makes it necessary to strictly limit the size of the aperture diaphragm, which in turn leads to a sharp increase in the effects of diffraction scattering of the electronic image. Therefore, one of the most important problems of modern electron microscopy with high and ultrahigh resolution is correction of the main types of axial aberrations of electronic lenses - third order spherical and second order axial chromatic aberrations. To date, significant progress has been made in the development of various types of aberration correctors that allow simultaneous elimination of spherical and chromatic aberrations of an objective lens [2-8]. However, the use of such correctors significantly complicates the electron-optical scheme of the lens, which in addition to the objective lens itself, should include additional elements - electron mirrors, multipole lenses, magnetic deflectors and other elements. Meanwhile, as shown in [9], under some conditions in electron mirrors the coefficients of spherical and (or) axial chromatic aberration can be equal to zero. In principle, such mirrors can be used as the objective of an electron microscope.

Figure 1 shows the electron-optical scheme of a transmission electron microscope with a mirror lens [9]. The optical axis of the mirror coincides with the optical axis of the electron microscope. To achieve high magnifications, the object plane is located close to the focal plane of the mirror. The electron microscope works on the principle of forming an image in a dark field. The electron beam 2 formed by the lighting system 1 falls on the object under study located on the table of objects 3 at an angle to the main optical axis of the microscope. With the appropriate choice of the specified angle, only those electrons will pass through the aperture diaphragm 6, which, due to interaction with the object, deviated from their original path. The other part of the electrons that have not experienced interaction with the object will be delayed by this diaphragm. The electrons 4 deviated from the initial path reflected from the electrostatic electron mirror - objective 5, will create an enlarged image of the object corrected from aberrations on the intermediate screen 7 . Further, the observation and image registration devices 8 will create an enlarged image of this intermediate image in the plane of the screen 9.

Calculations of the geometric and electrical parameters of a three-electrode mirror lens providing a magnified image of an object (in the range from 10 to 50 times) with a simultaneous elimination of two main types of aberrations - third order spherical and aperture chromatic aberrations - were performed in the energy range up to $500 \mathrm{keV}$, with full account for relativistic effects. This result opens up a perspective of a qualitative increase in the resolving power of an electron microscope without significant complication of its design - by using an electron mirror free of basic aberrations as an objective. The performed calculations are aimed at solving some problems of electron imaging for modern high-resolution electron microscopy.

E-mail: bimurzaev@mail.ru 


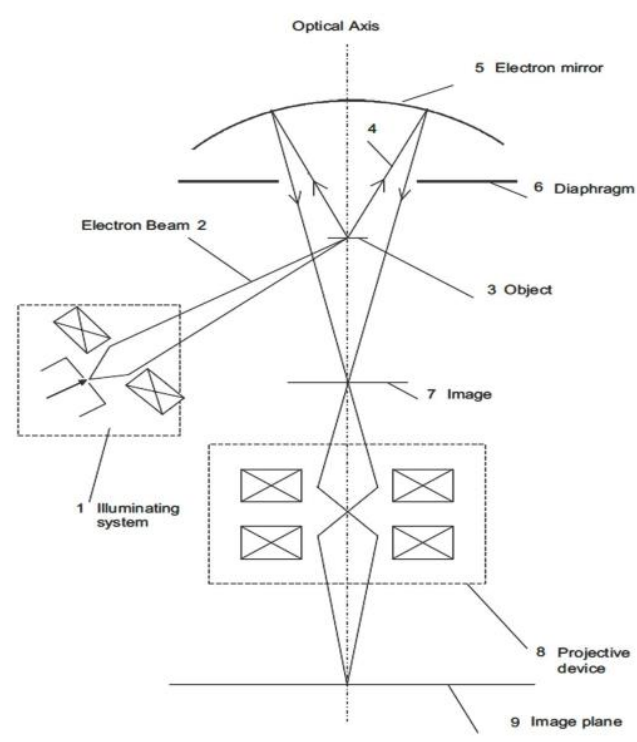

Figure 1. Scheme of electron microscope with a mirror lens

\section{References}

[1] P.W. Hawkes and E. Kasper. Principles of Electron Optics. Academic Press, 1989.

[2] O.L. Krivanek, P.D. Nellist, N. Dellby, M.F. Mur_tt, and Z. Szilagyi. Towards sub-0.5 A electron beams. Ultramicroscopy, 96(3): 229 - 237, 2003.

[3] P. Hartel, D. Preikszas, R. Spehr, H. Muller, and H. Rose. Mirror corrector for low-voltage electron microscopes. Advances in Imaging and Electron Physics, 120: 41--133. Elsevier, 2003.

[4] M. Haider, H. Rose, S. Uhlemann, E. Schwan, B. Kabius, and K. Urban. A spherical-aberrationcorrected $200 \mathrm{kv}$ transmission electron microscope. Ultramicroscopy, 75(1): 53--60, 1998.

[5] M. Haider, H. Muller, S. Uhlemann, J. Zach, U. Loebau, and R. Hoeschen. Prerequisites for a cc/cscorrected ultrahigh-resolution tem. Ultramicroscopy, 108(3): 167--178, 2008.

[6] H. Muller, S. Uhlemann, M. Haider, P. Hartel and J. Zach. Current and future aberration correctors for the improvement of resolution in electron microscopy. Trans. R. Soc. A., 367(1): 3665--3682, 2009.

[7] R.M. Tromp, J.B. Hannon, A.W. Ellis, W. Wan, A. Berghaus, and O. Schaff. A new aberrationcorrected, energy-filtered leem/peem instrument. i. Principles and design. Ultramicroscopy, 110 (7): 852$-861,2010$.

[8] R.M. Tromp, J.B. Hannon, W. Wan, A. Berghaus, and O. Schaff. A new aberration-corrected, energyfiltered leem/peem instrument. ii. Operation and results. Ultramicroscopy, 127: 25--39, 2013.[9] S.B. Bimurzaev, G.S. Serikbaeva and E.M. Yakushev. Electrostatic Mirror Objective with Eliminated Spherical and Axial Chromatic Aberrations. J. Electron Microscopy, 2003, 52(4): P.365-368. 